\title{
Construction of 3D Mandelbrot Set and Julia Set
}

\author{
Ankit Garg \\ Assistant Professor \\ Amity University, Haryana \\ Manesar, Gurgaon
}

\author{
Akshat Agrawal \\ Assistant Professor \\ Amity University, Haryana \\ Manesar, Gurgaon
}

\author{
Ashish Negi \\ Associate Professor \\ GBP, Engineering college and \\ Technology \\ Pauri, Garhwal
}

\begin{abstract}
Today Fractal geometry is completely new area of research in the field of computer science and engineering. It has wide range of applications. Fractals in nature are so complicated and irregular that it is hopeless to model them by simply using classical geometry objects. Benoit Mandelbrot, the father of fractal geometry, from his book The Fractal Geometry of Nature, 1982. This paper present construction of famous fractal images- Mandelbrot set and Julia set using 3D iterated function system which gives real look and feel of complex natural fractal images.
\end{abstract}

Keywords: Fractals, IFS, 3D images, 3D rendering, Mandelbrot set and Julia set, affine transformation.

\section{INTRODUCTION}

In computer graphics we use fractal functions to create complex objects. Objects appeared in nature can be described or created by using classical geometry. People believes that the objects in nature can be created or can be described by figures such as a lines, circles, conic sections, polygons, sphere, and quadratic surfaces and so on. Many objects in nature, which are complicated and irregular, cannot be modeled by classical geometry. In 1918, French mathematician Gaston Julia [1] investigated the iteration process of a complex function intensively and attained a Julia set, which is a landmark in the field of fractal geometry. The Julia set defines the boundary between prisoner set and escape set [2]. The prisoner set is a collection of points inside the Mandelbrot set and escape set is a collection of points outside the orbit of Mandelbrot set. Julia set is the place where all of the chaotic behavior of a complex function occurs [3]. Both the Mandelbrot and Julia Set fractals require the use of complex numbers to compute the basic Mandelbrot (or Julia) set one uses the equation $\mathrm{f}_{\mathrm{c}}(\mathrm{Z})=\mathrm{Z}^{\mathrm{n}}+\mathrm{C}$, where both $\mathrm{Z}$ and $\mathrm{C}$ are complex numbers. The object Mandelbrot set given by Mandelbrot in 1979 and its relative object Julia set have become a wide and enlite area of research due to their beauty and complexity of their nature [4].

Many authors have presented the paper on several "orbit traps" rendering methods to create the artistic fractal image. An orbit trap is a bounded area in the complex plane into which an orbiting point may fall [5]. Recently M.Rani and V. Kumar [6] introduced the superior Mandelbrot set using Mann iteration procedure.

"Clouds are not spheres, mountains are not cones, coastlines are not circles, and bark is not smooth, nor does lightning travel in a straight line. "(Benoit Mendalbrot). Man iteration and Ishikawa iteration process are generally used to approximate the fixed point. There is a lot of history behind Fractals even though the word fractal did not exist until the 1970s [7]. The Cantor Set discovered in 1872 by Georg Cantor [8], Sierpinski Gasket in 1916 by Wallow Sierpinski who created a triangle and a carpet, Koch Curves in 1904 by Helge Von Koch [9], Lévy C curve in 1938 by Paul Pierre Lévy have been known for quite some time [10]. The method of function iteration analyzes the sequences of numbers $\{X n\}$ generated by the formula $X_{n+1}=f\left(X_{n}\right)$ where $f$ is a complex function. The color of each point represents how quickly the values reach the escape point. Often black is used to show values that fail to escape before the iteration limit, and gradually brighter colors are used for points that escape. This gives a visual representation of how many cycles were required before reaching the escape condition. Points bounded to origin converge to a fixed point. Fixed points can be classified in three categories: Asymptotically stable: Means all nearby solutions converge to it.

Stable: Means all nearby solutions stay nearby.

Unstable: Means mostly all nearby solutions diverge away from the fixed point. When testing a point in a plane to see if it is part of the set, the initial value of $\mathrm{z}$ is always zero. This is so because zero is the critical point of the equation used to generate the set [11].Formally, the fractals are categorized in two types i.e. regular (geometric) and random fractals. Regular fractals consist of large and small structures that are exact copies of each other, except in size [12]. One of the more well known regular fractals is the Koch snowflake, which is made up of small triangles added to the sides of larger triangles to an infinite degree [12]. Random fractals are more apparent in nature as their small scale structures may differ in detail. It was this type of pattern that greatly influenced Mandelbrot, who gave these patterns the name fractal, from the Latin word fractus, which means a broken stone with an irregular surface. There are two important properties of fractals are self-similarity and dimension. There are typically four techniques to generate fractals: escape-time fractals, iterated function systems, random fractals, and strange attractors [13]. In functional dynamics, we have existence of two different types of points. Points that leave the interval after a finite number are in stable set of infinity. Points that never leave the interval after any number of iterations have bounded orbits. So, an orbit is bounded if there exists a positive real number, such that the modulus of every point in the orbit is less than this number.

The collection of points that are bounded, i.e. there exists $M$, such that $\left|\mathbf{Q}^{\mathbf{n}}(\mathbf{Z})\right|<=\mathbf{M}$, for all $\mathrm{n}$, is called as a prisoner set while the collection of points that are in the stable set of infinity is called the escape set.

\section{NATURAL PHENOMENA OF FRACTALS}

Many objects in the nature can be created by applying the concept of classical geometry like- lines, circles, conic sections, polygons, spheres, quadratic surface and so on. There are various objects of nature which cannot be modeled by applying Euclidean geometry, hence there is need to deal 
with such complicated and irregular object which can only be constructed by fractal geometry.

To generate such complicated object iteration process is required which is called iterated function system.

The main property in every fractal object is self similarity. Upon magnification of a Fractal, we can find subsets of it that look like the whole figure. If we zoom on a picture of a mountain again and again we still see a mountain. This is the self similarity of fractal.

\subsection{Mandelbrot set}

In a complex plane Mandelbrot set is the set of values of $c$ for which the orbit of 0 under iteration of the complex quadratic polynomial

$$
z_{n+1}=z_{n}^{2}+c
$$

remains bounded. Suppose the initial value of $Z_{0}=0$, if this function iterates again and again with a large value of $Z_{n}$, the number $C$ may or may not be part of Mandelbrot set.

This function with $c=1$ gives the sequence $0,1,2,5,26, \ldots$, which tends to infinity. As this sequence is unbounded, Hence $C$ with value 1 is not an element of the Mandelbrot set.

If the value of , $C$ is -1 this function after few iteration gives the sequence $0,-1,0,-1,0, \ldots$, which is bounded, in this case $C$ belongs to the Mandelbrot set. A time series plot of the orbit often gives more information about the fate of the orbits. Thus different points can have very different behavior under iteration by this single function $z_{n+1}=z_{n}^{2}+c$.

\subsection{Classification of fractal image}

Fractal image can be constructed as a two-dimensional array of pixels and each pixel in image can be represented by a pair of coordinates $(\mathrm{X}, \mathrm{Y})$. The complex quantity $\mathrm{Z}$ and $\mathrm{C}$ can be represented as[18]:

$$
\begin{aligned}
& Z=Z_{x}+i Z_{x} \\
& C=C_{x}+i C_{y}
\end{aligned}
$$

Where $i=\sqrt{-1}$ and $\mathrm{Zx}$ and $\mathrm{Cx}$ are the real part and $\mathrm{Zy}$ and $C y$ are the imaginary part of $Z$ and $C$ respectively. Every pixel value is associated with $\left(C_{x}, C_{Y}\right)$ or $\left(Z_{x}, Z_{y}\right)$.

Fractal image can be classified as follows [18]:

Z-plane fractal: Where $(X, Y)$ is a function of $\left(Z_{x}, Z_{Y}\right)$

C-plane fractal: Where $(X, Y)$ is a function of $\left(C_{X}, C_{y}\right)$.

\section{ITERATING FUNCTIONS WITH COMPLEX NUMBERS AND THE MANDELBROT SET}

The basic principle of generating fractals employs the iterative formula $(Z)=Z^{2}+$ c. suppose the initial value of $Z$ is 0 . By putting the value of $Z$ compute $0^{2}+\mathbf{c}$ which give $\mathbf{c}$. After repeating this process we get $\left(\mathbf{c}^{2}+\mathbf{c}\right)^{2}+\mathbf{c}$. And so forth. The list of complex number will be generated in this way. If these complex numbers (called the orbit of $\mathbf{0}$ ) get larger and larger (or further and further away from the origin), then choice of $\mathbf{c}$ is NOT in the Mandelbrot set. But if this is not the case (the orbit stays "bounded"), then $\mathbf{c}$ IS in the Mandelbrot set [14].

The picture of Mandelbrot can be painted according to the following rule: Color a c-value black if $\mathrm{c}$ does not escape to infinity, it means $\mathrm{C}$ lie within the Mandelbrot set. Color a cvalue a different color if the orbit escapes to infinity. Few iteration are required for the orbit of 0 under iteration of $x^{2}+c$ to become far from the origin. Red points are followed in order by orange, yellow, green, blue, indigo, and violet. That is we use the colors drawn from the light spectrum for points that are not in the Mandelbrot set. The important thing is: red points escape fastest, while violet points take the most iteration to go far away from the origin [14].

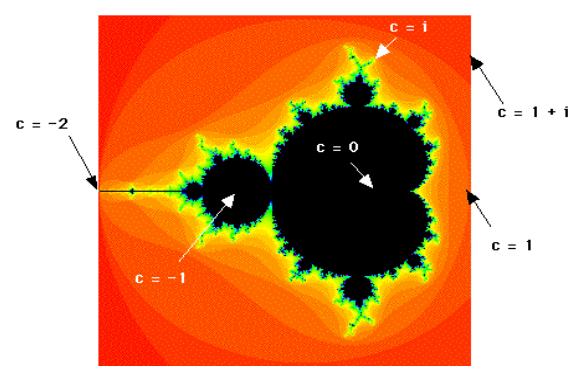

Fig 1: orbit of mendelbrot set

The filled Julia set for $\mathbf{x}^{2}+\mathbf{c}$ is the collection of all seeds whose orbit does not escape to infinity under iteration of $\mathbf{x}^{2}+\mathbf{c}$. Thus there is a different filled Julia set for each c-value.

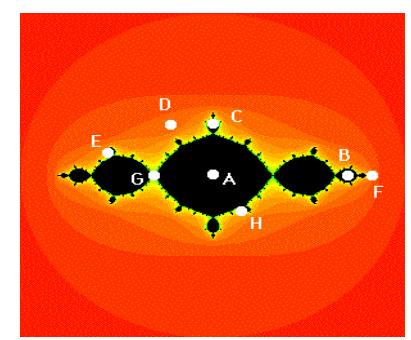

Fig 2: Corresponding Julia set of a seed inside the Mandelbrot set.

\section{RELATIONSHIP BETWEEN THE MANDELBROT SET AND FILLED JULIA SETS}

Mandelbrot is a picture of the complex plane. Every point inside the Mandelbrot set represents a value for which the orbit of $\mathbf{0}$ does not escape under iteration of $\mathbf{x}^{2}+\mathbf{c}$. The shape of Julia set is depending upon the value of $\mathrm{c}$ which may be inside the Mandelbrot set or may be outside the Mandelbrot set.

If the value of $\mathbf{c}$ which is a complex number lies in the Mandelbrot set, then the corresponding filled Julia set is connected, meaning it is just one piece. If the value of $\mathbf{c}$ lies outside the Mandelbrot set, then the filled Julia set shatters into infinitely many pieces (what is known technically as a "Cantor set" or, more popularly, "fractal dust").

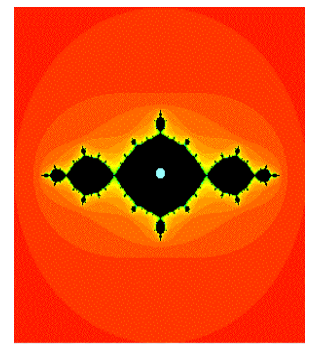

(a)

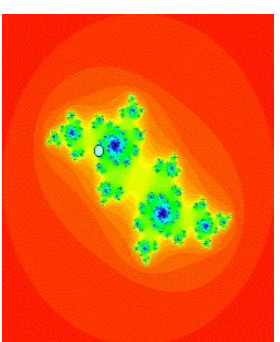

(b)
Fig 3: Julia set (a) connected Julia set (b) Disconnected Julia set 


\section{ITERATED FUNCTION SYSTEMS FOR 2D MANDELBROT SET AND JULIA SET}

In the mid 1980's, IFS's become very popular. It was Barnsley and his co-workers at Georgia Institute of Technology who first noticed the potential of IFS for applications in computer graphics. Barnsley and Sloan advertised in popular science magazines the incredible power of IFS for compressing color images at rates of over 10,000 to 1.Following are two algorithms which comes under IFS. Transformation used to generate fractals should be contractive. By applying contractive transformation on an image will create an attractor which is fractal with the same structure at any level of detail.

This is the basic property of fractal. Transformation should be contractive otherwise the attractor will spread out to be infinite size. Different attractors can be generated by applying different affine transformation over the same image. Each iteration under iterated function system provides reduced copy of original one. The multiple copies of original image is nothing but the result of affine transformation.

An iterated function system with probabilities consists of an IFS

$$
\left\{\mathrm{X} ; w_{1}, w_{2}, \ldots, w_{\mathrm{N}}\right\}
$$

Together with an ordered set of numbers $\left\{p_{1}, p_{2}, \ldots, p_{\mathrm{N}}\right\}$, such that

$$
\begin{gathered}
p_{1}+p_{2}+p_{3}+\ldots+p_{\mathrm{N}}=1 \text { and } p_{i}>0 \\
\text { for } i=1,2, \ldots, N .
\end{gathered}
$$

The probability $p_{i}$ is associated with the transformation $w_{i}$. The nomenclature IFS with probabilities may be used as an abbreviation. The full notation for such an IFS is

$$
\left\{\mathbf{X} ; w_{1}, w_{2}, \ldots, w_{\mathrm{N}} ; p_{1}, p_{2}, \ldots, p_{\mathrm{N}}\right\} \text {. }
$$

The probabilities are related to the measure theory of IFS attractors, and play a role in the computation of images of the attractor of an IFS attractor using the random iteration algorithm.

\section{ITERATED FUNCTION SYSTEMS FOR 3D MANDELBROT SET AND \\ JULIA SET}

The parameters of an iterated function system to generate fractal image are already defined by the formula itself. But some parameters still remain to be specified. The other parameters are:

- Iteration counter

It specifies the number of point that will be iterated.

- Color mode

It specifies the color to paint the calculated point using IFS.

a) Transformation: Colors each point according to the transformation, which have lead to it.

b) Probability: Colors each point according to the probability, i.e. according to the previous random number.

c) Measure: Colors each pixel in the window according to how often a point hits the pixel.

Following affine matrix is required for 3D fractal image.

$\begin{array}{cccc}\mathrm{a} & \mathrm{b} & \mathrm{c} & \mathrm{d} \\ \mathrm{e} & \mathrm{f} & \mathrm{g} & \mathrm{h} \\ \mathrm{j} & \mathrm{k} & \mathrm{l} & \mathrm{m}\end{array}$

$$
\begin{array}{llll}
0 & 0 & 0 & 1
\end{array}
$$

2D fractal can be generated in 3D environment by simply putting $\mathrm{j}=\mathrm{k}=\mathrm{l}=\mathrm{m}=\mathrm{c}=\mathrm{g}=0$ and taking remaining values $\mathrm{a}, \mathrm{b}, \mathrm{d}, \mathrm{e}, \mathrm{f}$ according to the $2 \mathrm{D}$ affine transformation.

\section{CONSTRUCTION OF FERN IN 3D ENVIRONMENT}

To generated fern like fractal object it is required to have four functions.

One function is used to make stem of fern. To form a straight line this function project all the points on $\mathrm{T}$ axis.

Two another functions are required to place leaves of fern on the left and right directions. To place leaves in right and left directions rotation, scaling and translation transformations are required. Last function represents the rest of fern, and transforms it by shrinking it slightly. To make a fern in 3D rotation about $\mathrm{Y}$ axis and $\mathrm{Z}$ axis is required.

Rotation matrix about $\mathrm{Y}$ axis in $3 \mathrm{D}$ can be represented as follows.

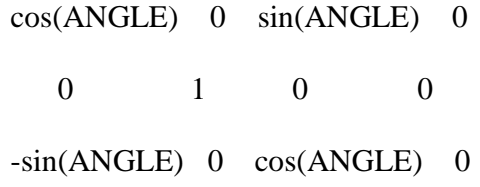

Here

is

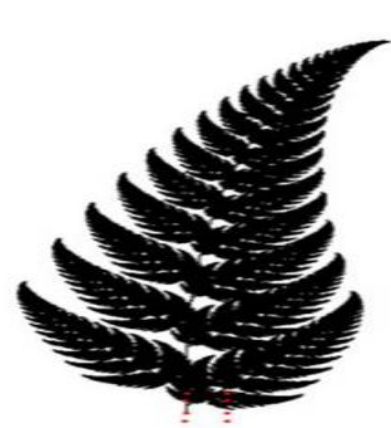

ANGLE angle of rotation.

Fig 4: Image of Fern in 2D

The 3D version of this image seems very bushy and difficult to see what is happening. To generate clear image it is require to have a system to keep the $3 \mathrm{D}$ rotation while restricting the rotation within leaves. This can be accomplished for each leaf, project the fern to the XY plane and then rotate it.

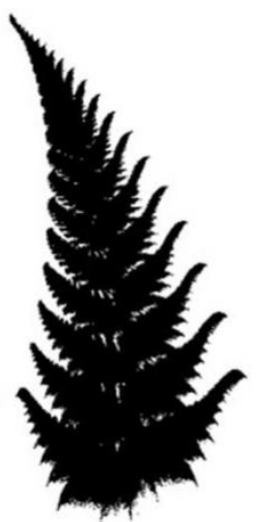


Fig 5: Image of fern in 3D

\section{EXPERIMENTAL RESULT}

All the results are generated in $3 \mathrm{D}$ image rendering software in MATLAB.

\subsection{Generation of Mandelbrot set}

This section depicts the images generated by using IFS which is based on defined IFS parameters. The resulting 3D Mandelbrot set can be rendered by using MATLAB 3D image rendering software.

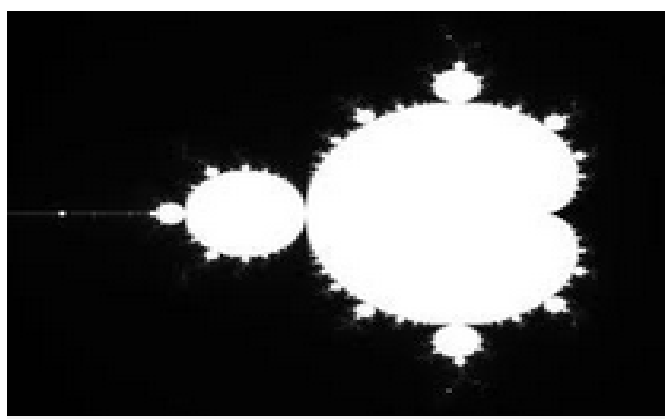

Fig 6: 2D Mandelbrot set

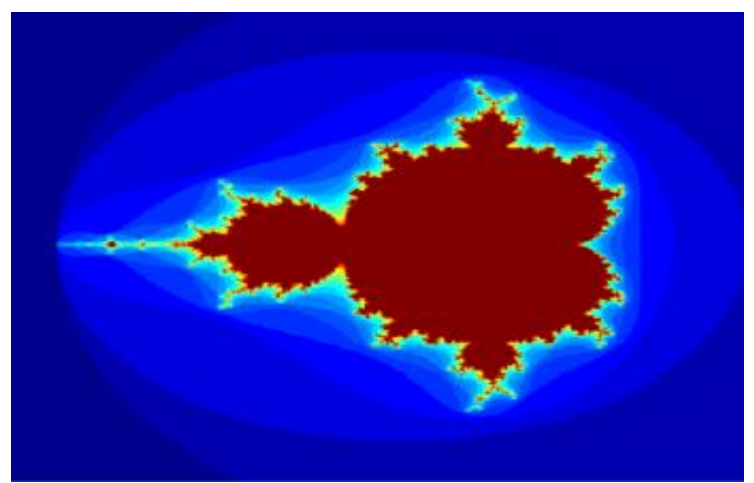

Fig 7: 3D Mandelbrot Set generated using 24 iterations, and $(X, Y)$ ranges as $[-2.1,1]$ and $[-1.5,1.5]$

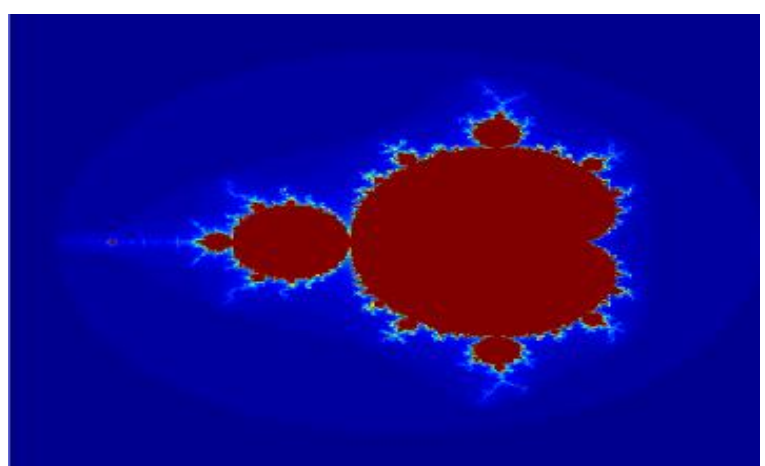

Fig 8: 3D Mandelbrot Set generated using 100 iterations, and $(X, Y)$ ranges as $[-2.1,1]$ and $[-1.5,1.5]$

After iterating 100 times a different 3D image of the

Mandelbrot Set is obtained, as shown in Fig. 9

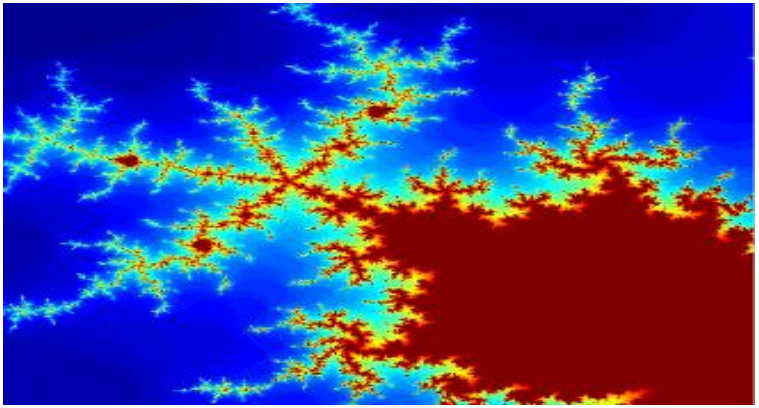

Fig 9: 3D Mandelbrot Set generated using 100 iterations, and $(X, Y)$ ranges as $[-1.23,-1.19]$ and $[0.12,0.17]$

\subsection{Generation of the 3D Julia Set}

Different structure of Julia set can be obtained by changing number of iterations and random co-efficient $\mathrm{c}$. The resulting Julia set can be rendered as 3D Julia set by using MATLAB 3D Image Rendering software. Fig. 10 shows the initial 2D image of the Julia Set. Figures 11-13 show the outputs of the same based on 25 and 100 iterations.

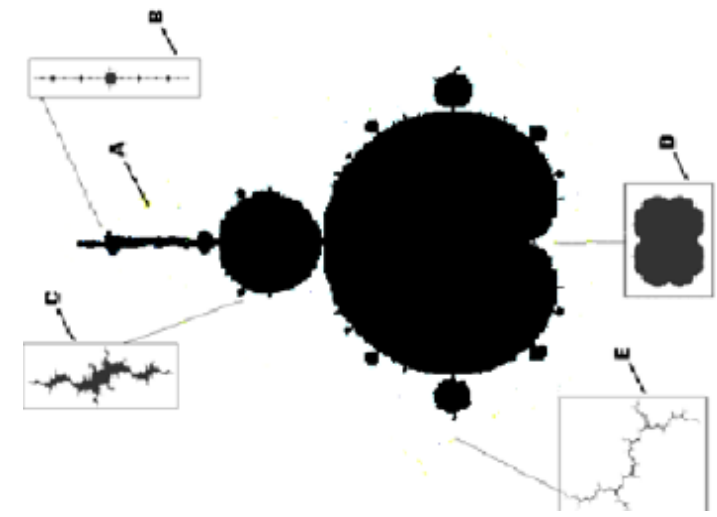

Fig 10: 2D Julia Set - Initial Image - The sub-images A, B, $\mathrm{C}, \mathrm{D}, \mathrm{E}$ are the derived 2D Julia Sets obtained by an iterative zoom-in process of the $2 \mathrm{D}$ version of Mandelbrot Set.

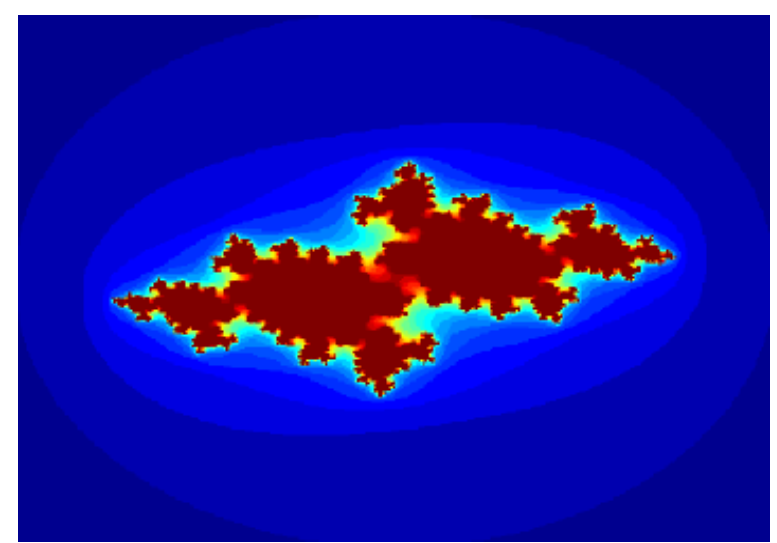

Fig 11: 3D Julia Set generated using 25 iterations, and $(X$, Y) ranges as $[-1.9,1.9]$ and $[-1.9,1.9]$ and $\mathrm{c}=\mathbf{- 0 . 6 - 0 . 2} *$ iter (iter $=1$ to 25). 


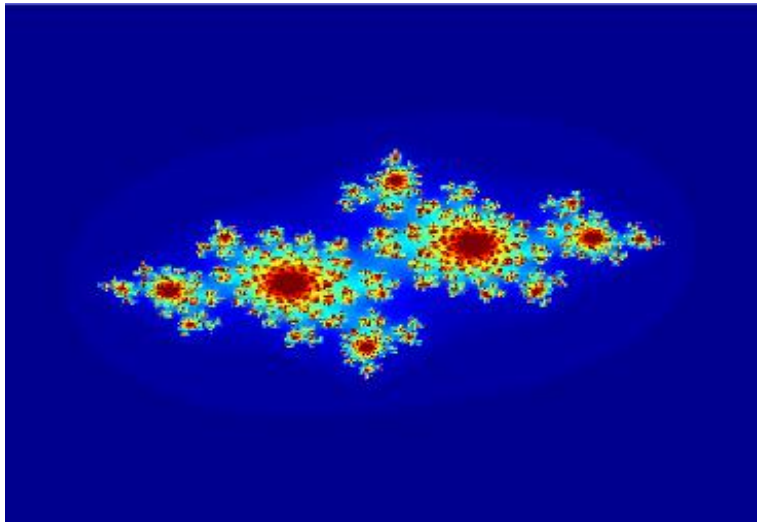

Fig. 12: 3D Julia Set generated using 100 iterations, and $(\mathrm{X}, \mathrm{Y})$ ranges as $[-1.9,1.9]$ and $[-1.9,1.9]$ and $\mathrm{c}=\mathbf{- 0 . 6}$ $0.2 *$ iter $($ iter $=1$ to 100$)$

After iterating 100 times a 3D image of the Julia Set is obtained, as shown in Fig.13. Here we change the value of c to be calculated as:

$$
\mathrm{c}=-0.83-0.20 * \text { iter }(\text { iter }=1 \text { to } 100) \text {. }
$$

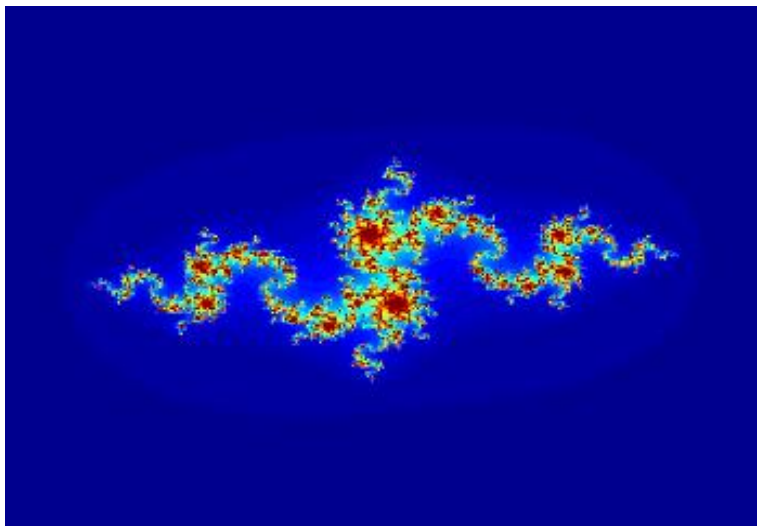

Fig. 13: 3D Julia Set generated using 100 iterations, and $(\mathrm{X}, \mathrm{Y})$ ranges as $[-1.9,1.9]$ and $[-1.9,1.9]$ and $\mathrm{c}=\mathbf{- 0 . 8 3}$ $0.20 *$ iter (iter $=1$ to 100$)$.

\section{CONCLUSION}

All the experiments depicted above shows the construction of 3D fractal images Mandelbrot set and julia set. The 3D version of Mandelbrot set and Julia set in 2D look like same. By using 3D image rendering software in MATLAB 3D version of $2 \mathrm{D}$ fractal images can be constructed. The results presented in this paper are based on number of iteration involve, 3D co-ordinates range, and the choice of calculating the co-efficient $c$. This enables generation of more beautiful images and in higher dimensions too.

\section{ACKNOWLEDGEMENTS}

I thank my guide Dr. Asish Negi, Associate professor in Department of Computer Science and Engineering, Govind Ballabh pant Engineering College, Pauri Garhwal for providing me an opportunity and resource to carry out this work. I am also thankful to NPTEL funded by ministry of HRD government of india to carry out this work which provides E-learning through online Web and Video courses in Engineering, Science and humanities streams.

\section{REFERENCES}

[1] B. B. Mandelbrot, The Fractal Geometry of Nature (W.H. Freeman and Company, 1982).

[2] Chauhan Y. S. Rana R,and Negi A, "Mandel-Bar Sets of Inverse Complex Function", International Journal of Computer Applications (0975-8887) Volume 9- No.2, November 2010

[3] Devaney RL. A first course in chaotic dynamical systems: theory and experiment. CO: Westview Press; 1992.

[4] [4] Yashwant s chauhan, Rajeshri rana, Ashish negi, "Mandel-Bar sets of Inverse Complex Function", International Journal of computer applications(09758887), Vol 9-No.2, Nov-2010.

[5] Yashwant s chauhan, Rajeshri rana, Ashish negi, "New Julia sets of Ishikawa Iterates", International Journal of computer applications(0975-8887), Vol 7-No.13, oct2010 .

[6] M.Rani and V.Kumar, "Superior Mandelbrot set". J korea Soc, Math, edu, series, Dresearch in maths, Edu, no. 4,8(2004), 279-291.

[7] Barnsley, M., 1988, "Fractals Everywhere (San Diego: Academic Press, Inc).

[8] George Cantor "On the Power of Perfect Sets of Points in Classics on Fractals" (Westview Press, 2004) pp.1123.

[9] [ONLINE AVAILABLE]: Bulusu Rama\#, Jibitesh Mishra, Generation of 3D Fractal Images for Mandelbrot and Julia Sets

[10] M. F. Barnsley and R. Hawley, Fractals Everywhere (Boston: Academic Press Professional, 1993).

[11] http://math.bu.edu/DYSYS/explorer/tour1.html

[12] http://library.thinkquest.org/26242/full/ 